\title{
Osteomielitis de tibia proximal por Salmonella grupo $B$ en un paciente joven inmunocompetente.
}

\author{
DOI: http//dx.doi.org/10.37315/SOTOCAV20212855622 \\ DARÁs BALLESTER $A^{1}$, BONILLA ENSEÑAT $\mathrm{F}^{1}$, DE LA CALVA CEINOS $\mathrm{C}^{2}$, FUERTES LANZUELA $\mathbf{M}^{3}$. \\ 1. SERVICIO DE CIRUGÍA ORTOPÉDICA Y TRAUMATOLOGÍA, HOSPITAL UNIVERSITARIO Y POLITÉCNICO LA FE, VALENCIA, ESPAÑA \\ 2. UNIDAD DE TUMORES Y SÉPTICOS, SERVICIO DE CIRUGÍA ORTOPÉDICA Y TRAUMATOLOGÍA, HOSPITAL UNIVERSITARIO Y POLITÉCNICO LA FE, \\ VALENCIA, ESPAÑA \\ 3. SERVICIO DE CIRUGÍA ORTOPÉDICA Y TRAUMATOLOGÍA, HOSPITAL LLUÍS ALCANYÍS, XÀTIVA, ESPAÑA
}

\begin{abstract}
Resumen.
Nuestro objetivo es presentar un caso de osteomielitis de tibia proximal por Salmonella, infrecuente en la práctica clínica, así como analizar los procedimientos llevados a cabo para su tratamiento, y el diagnóstico diferencial de la lesión. Caso clínico: Varón de 13 años remitido desde otro centro a Oncología Infantil de nuestro centro por sospecha de tumor óseo en tibia. El paciente refería dolor en la rodilla de dos semanas de evolución asociado a fiebre. En las radiografías realizadas en su centro de referencia se observaba una lesión lítica en la metáfisis proximal de la tibia, que motivó la realización de una Resonancia Magnética (RM). Tras revisarse las imágenes en nuestro centro, se descartó la naturaleza neoplásica de la lesión y el diagnóstico de sospecha fue de una osteomielitis, tras lo cual el paciente se intervino, realizándose una corticotomía y vaciamiento del absceso e iniciando tratamiento antibiótico intravenoso. La evolución postoperatoria fue favorable y en el cultivo del material extraído creció Salmonella grupo B. El paciente fue dado de alta con antibiótico oral, siendo dado de alta definitivamente a los dos años de la intervención. No obstante, 3 años después del alta, presentó nuevamente un cuadro similar. Tras realizar pruebas de imagen, se observaron dos lesiones líticas multiloculadas en tibia proximal con reacción inflamatoria perilesional, sugestivas de recidiva de la Osteomielitis, por lo que volvió a intervenirse, realizándose una ventana ósea y legrado, y rellenando la cavidad con Sulfato tricálcico y antibiótico. Los cultivos tomados volvieron a ser positivos para Salmonella B. Tras la normalización clínico-analítica del paciente, fue dado de alta con antibioterapia oral, y se le dio el alta definitivamente a los dos años de la intervención. Conclusiones: La Osteomielitis por Salmonella en pacientes inmunocompetentes es una patología infrecuente que puede, además, simular una lesión neoplásica si se encuentra en una localización atípica como las metáfisis. El diagnóstico debe ser clínico, radiológico y analítico, aunque el diagnóstico definitivo será mediante cultivo. El tratamiento definitivo es el legrado y relleno de la lesión asociado a antibioterapia, y es necesario tener en cuenta que la lesión puede recidivar.
\end{abstract}

Palabras clave: Salmonella; osteomyelitis; metaphyseal osteolytic lesions; inmunocompetent

\section{Summary.}

Our objective is to present a case of proximal tibial osteomyelitis due to Salmonella, which is infrequent in clinical practice, as well as to analyze the procedures carried out for its treatment, and the differential diagnosis of the lesion. Clinical case: A 13-year-old male referred from another Hospital to our Hospital Children's Oncology due to a suspicion of a bone tumor in the tibia. The patient reported two-week history of knee pain associated with fever. In the radiographs taken at his center, a lytic lesion was observed in the proximal metaphysis of the tibia, which led to the performance of a Magnetic Resonance Imaging (MRI). After reviewing the MRI images in our center, the neoplastic nature of the lesion was ruled out and the suspected diagnosis was an Osteomyelitis, after which the patient underwent surgery, being performed a corticotomy and emptying of the abscess and initiating intravenous antibiotic treatment. The patient had an unremarkable postoperative course and in the culture of the extracted material Salmonella group B was obtained. The patient was controlled with oral antibiotic, and was definitively discharged two years after the intervention. However, 3 years after that, he came to Hospital with similar symptoms. After performing some imaging tests, two multiloculated lytic lesions were observed in the proximal tibia with a perilesional inflammatory reaction, suggestive of recurrence of Osteomyelitis, for which he underwent surgery again, performing a bone window and curettage, and filling the cavity with tricalcium sulfate and antibiotic. The cultures taken were again positive for Salmonella B. After the patient's clinical and analytical progress was correct, he was treated with oral antibiotics, and was definitively discharged two years after the intervention. Conclusion: Salmonella osteomyelitis in immunocompetent patients is a rare pathology that can simulate a neoplastic lesion if it is found in an atypical location such as metaphysis. The diagnosis must be clinical, radiological and analytical, although the definitive diagnosis will be achieved by cultures results. The definitive treatment is curettage and filling the lesion, associated with antibiotic therapy, and it is necessary to take into account that the lesion may recur.

Correspondencia: Álvaro Darás Ballester E-mail:

daras.alvaro@gmail.com 


\section{Introducción}

La osteomielitis por Salmonella es una patología rara, suponiendo solamente un $0,8 \%$ de todas las infecciones por Salmonella, y un $0,45 \%$ del total de osteomielitis diagnosticadas $^{1-6}$. Cuando se produce, frecuentemente afecta a pacientes con anemia de células falciformes o inmunocomprometidos $^{1-5}$. No obstante, es una entidad extremadamente rara en pacientes inmunocompetentes, y acerca de la cual existe muy poca literatura ${ }^{1-6}$

Además, este tipo de osteomielitis se observan predominantemente en la diáfisis de huesos largos y las vértebras, siendo infrecuente su localización metafisaria ${ }^{1-4}$.

En este artículo presentamos un caso de osteomielitis de tibia proximal por Salmonella $B$, en un paciente inmunocompetente, cuyo diagnóstico de sospecha inicial fue de patología oncológica, debido al aspecto osteolítico de las lesiones observadas en las pruebas de imagen, y a la localización metafisaria de las mismas.

El objetivo del artículo es exponer un caso infrecuente en la práctica diaria, así como describir el manejo de la misma, el diagnóstico diferencial, y los procedimientos terapéuticos llevados a cabo.

\section{Caso Clínico}

Varón de 13 años que es trasladado desde otro hospital al servicio de Oncología Infantil de nuestro centro por una sospecha de tumor óseo en tibia proximal izquierda. El paciente debutó con un cuadro gripal asociado a fiebre de $39,5^{\circ} \mathrm{C}$ de varios días de evolución. Poco a poco fueron desapareciendo las mialgias hasta quedar únicamente dolor en la rodilla izquierda que producía limitación funcional, le despertaba por la noche, y cedía con Paracetamol. En su hospital de referencia le realizaron radiografías anteroposterior (AP) y lateral de la rodilla afectada, en las que se podía apreciar una lesión en la zona metafisaria proximal medial de la tibia (Fig. 1), que motivó el ingreso del paciente para ampliar estudios. Durante el ingreso se le realizó una Resonancia Magnética (RM) en la cual se observaba una lesión osteolítica con importante componente inflamatorio perilesional y de partes blandas asociado (Fig. 2), planteando como primer diagnóstico de sospecha un Tumor de Células Gigantes (TCG).

A su ingreso en nuestro hospital, es valorado por el servicio de Oncología Infantil, que, tras revisar las imágenes conjuntamente con el servicio de Radiología Infantil, concluyen que los estudios de imagen excluyen una lesión maligna y son compatibles con una osteomielitis (absceso de Brodie), por lo que el paciente se intervino por parte de la Unidad de Sépticos del Servicio de Traumatología de nuestro Hospital, realizando una corticomía y vaciamiento del absceso, tomando muestras del tejido para cultivo bacteriológico y de micobacterias. Tras la toma de muestras, se inició tratamiento antibiótico con Cefuroxima iv durante 10

días. A los 4 días de la intervención, en el cultivo bacteriológico crece Salmonella grupo B.

a)

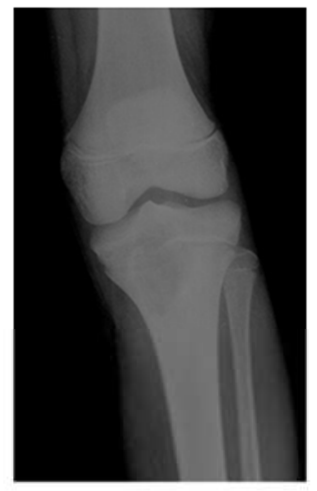

b)

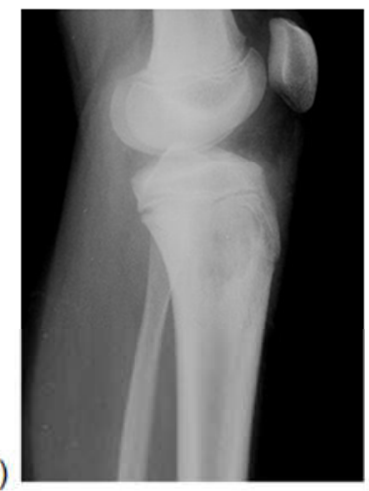

Figura 1: Radiografías iniciales AP (a) y lateral (b) de rodilla izquierda en las que se aprecia una imagen osteolítica en la metáfisis proximal de la tibia.

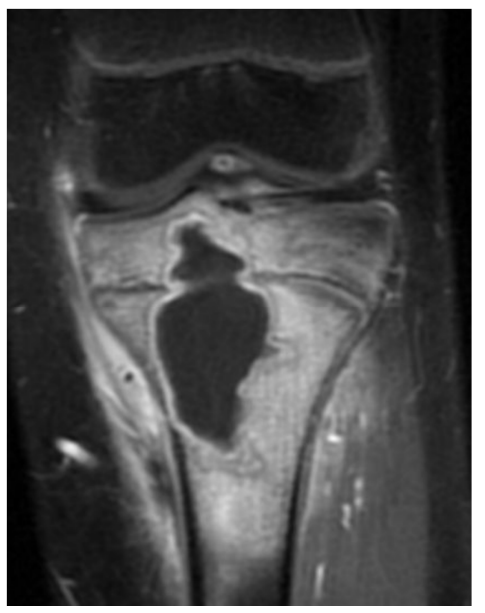

Figura 2: Imagen de resonancia magnética en la que se aprecia la lesión osteolítica en tibia proximal con importante componente inflamatorio perilesional y de partes blandas.

Dada la buena evolución clínica, los controles radiográficos correctos (Fig. 3) y la normalización de parámetros analíticos, el paciente fue dado de alta a los 10 días completando el tratamiento antibiótico con Ciprofloxacino oral y seguimiento en consultas externas. 

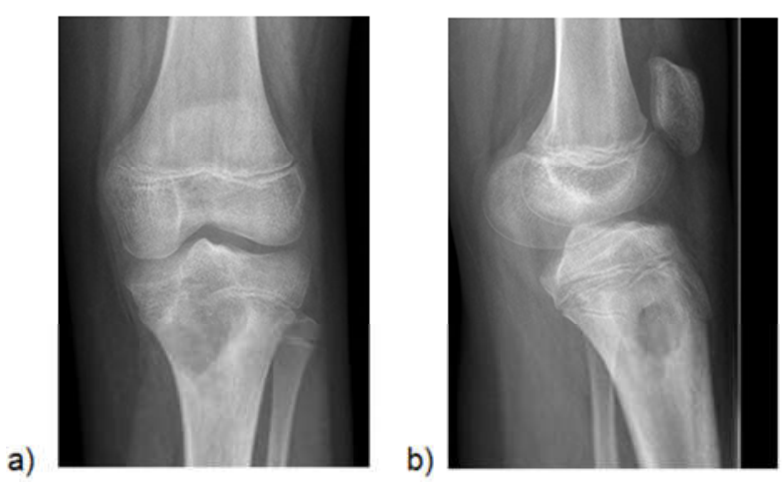

Figura 3: Radiografías anteroposterior (a) y lateral (b) de control posquirúrgico tras el vaciamiento del absceso.

En los controles en consultas externas el paciente presenta una buena evolución, iniciando actividad deportiva a los 3 meses de la intervención, apareciendo una mínima dismetría por hipercrecimiento de la tibia izquierda, y presentando un relleno completo de la lesión al año (Fig. 4), siendo dado de alta a los 20 meses de la intervención.

a)

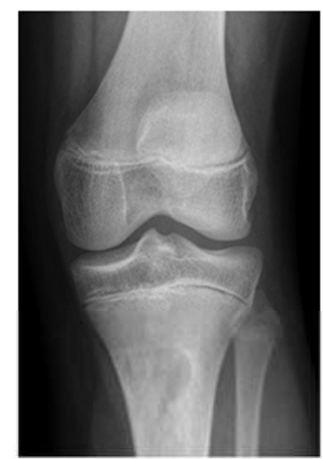

b)

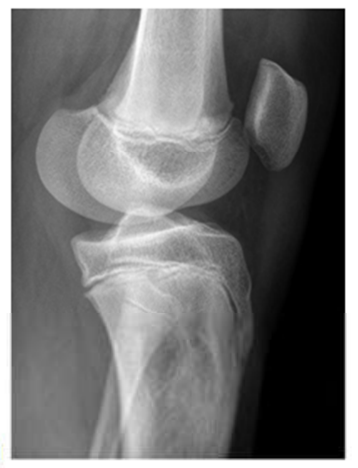

Figura 4: Radiografías de control anteroposterior (a) y lateral (b) al año de la intervención donde se aprecia un relleno prácticamente completo de la cavidad.

No obstante, dos años después del alta de consultas externas, con 18 años de edad, el paciente vuelve a ser derivado desde su Hospital de referencia por una sospecha de reagudización del episodio de osteomielitis en tibia proximal izquierda. Según refiere el paciente, aproximadamente un mes antes inicia un cuadro de dolor en la zona metafisaria proximal de la tibia, que se acompaña de picos febriles de $39^{\circ} \mathrm{C}$ y sensación distérmica. Además, refiere un aumento de temperatura en dicha zona.

En su hospital de referencia, previamente a remitirlo a nuestro Centro, le realizan un TC de rodilla, en el que se visualizan dos lesiones líticas multiloculadas en la zona metafisaria proximal de la tibia, alcanzando una de ellas la cortical medial de la tibia y generando reacción perióstica y una masa de partes blandas asociada, sin visualizarse trayectos fistulosos sugestivos de cloacas (Fig. 5), todo ello sugestivo de una reagudización de la Osteomielitis. a)

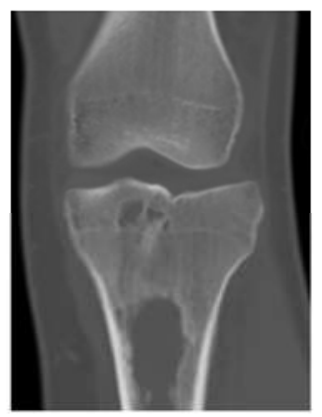

b)

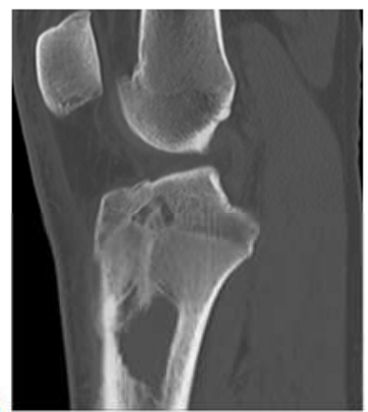

Figura 5: Cortes de TC en los que se aprecian dos lesiones líticas multiloculadas en la zona metafisaria proximal de la tibia, sugestivas de reagudización de la osteomielitis.

En nuestro centro de le realiza una RM de la rodilla izquierda, con hallazgos sugestivos de osteomielitis crónica epifisiometafisaria que asocia un absceso de Brodie polilobulado, reacción inflamatoria subperióstica y extenso edema de partes blandas (Fig. 6), también se observa derrame sinovial intraarticular compatible con artritis séptica incipiente.
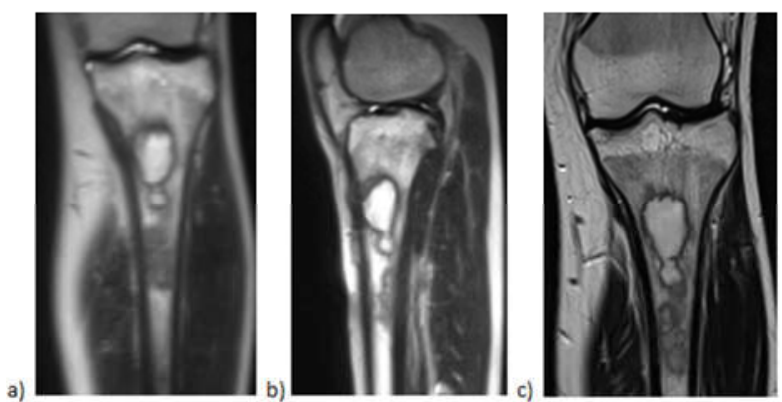

Figura 6: Hallazgos de RM sugestivos de reagudización de osteomielitis crónica de tibia proximal, que presenta un absceso de Brodie polilobulado asociado a reacción inflamatoria subperióstica y de partes blandas.

Tras los resultados de la RM, el paciente fue intervenido por la Unidad de Sépticos de nuestro hospital, tomando nuevas muestras para cultivo bacteriológico y Anatomía Patológica, realizando una nueva ventana ósea, con legrado y desbridamiento extenso bajo fluoroscopia (Fig. 7), y rellenando la cavidad con Sulfato Tricálcico asociado a Vancomicina y Gentamicina. 


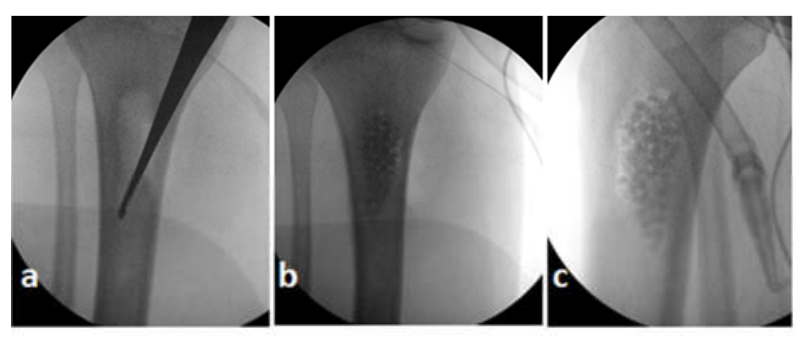

Figura 7: Imágenes de fluoroscopia en las que se observa cómo se realiza el legrado y desbridamiento extenso de la lesión, y se rellena la cavidad con Sulfato Tricálcico asociado a Gentamicina y Vancomicina.

Durante el postoperatorio, el paciente siguió con antibioterapia iv durante 10 días (Cefuroxima iv), siendo dado de alta tras la normalización de parámetros analíticos y la mejoría clínica. En todas muestras de cultivo intraoperatorio se aísla Salmonella grupo B.

Los controles en consultas externas fueron satisfactorios, manteniendo descarga del miembro durante 6 semanas, y permitiendo el inicio de actividades deportivas a las 12 semanas, tras observarse en radiografías cómo se va reabsorbiendo el Sulfato Tricálcico y se va rellenando la cavidad (Fig. 8). Finalmente, el paciente fue dado de alta a los 2 años de la intervención, completamente asintomático, y con los controles radiográficos satisfactorios (Fig. 9).

a)
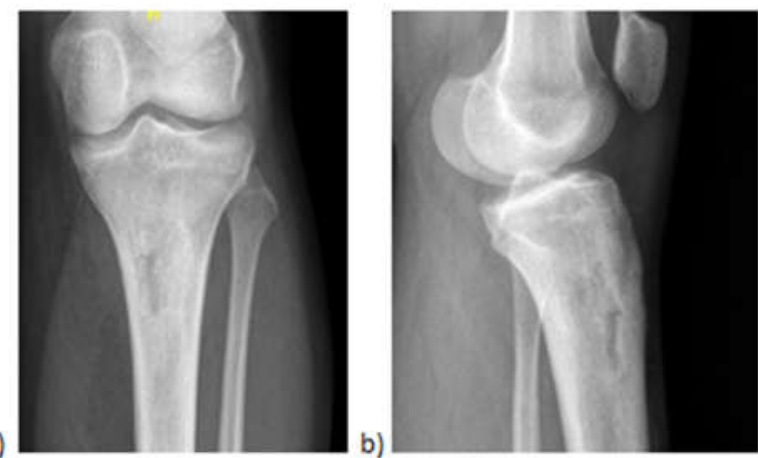

Figura 8: Controles radiográficos a las 12 semanas de la intervención en los que se observa cómo va reabsorbiéndose el sulfato tricáclico y se va rellenando la cavidad de hueso.

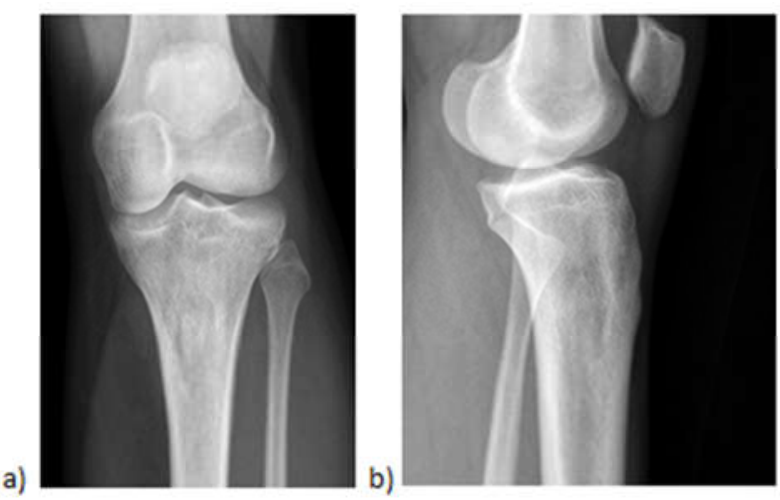

Figura 9: Controles radiográficos a los 2 años de la intervención, en los que se observa que la cavidad ósea se ha rellenado por completo.

\section{Discusión}

Las lesiones osteolíticas de los huesos largos ocurren con mayor frecuencia en la zona metafisaria, predominantemente alrededor de la rodilla. Cuando observamos lesiones de estas características, una de las primeras causas a descartar son lesiones tumorales, ya sean primarias o metastásicas. No obstante, en ocasiones, cuadros mucho menos frecuentes como la osteomielitis por Salmonella pueden dar imágenes similares ${ }^{1,2,5}$.

Dentro de los cuadros que puede causar la Salmonella, la afectación ósea es uno de los menos frecuentes, y suelen aparecer en pacientes inmunocomprometidos, o con patologías como hemoglobinopatías o anemia de células falciformes, pero raramente se observan en pacientes inmunocompetentes $^{1-6}$.

Además, mientras que las lesiones tumorales tienen una localización predominantemente metafisaria, las osteomielitis por Salmonella afectan típicamente a la diáfisis de los huesos largos, sobre todo el fémur y el húmero, $\mathrm{y}$ a las vértebras ${ }^{1-5}$.

Es destacable que no todos los pacientes afectos de una osteomielitis por Salmonella presentan una historia previa de cuadros diarreicos o cultivos positivos, es posible que los bacilos queden en un estado de quiescencia en el sistema retículo-endotelial y se reactiven con descensos de la respuesta inmunitaria del paciente ${ }^{1}$. De hecho, pueden pasar meses o incluso años hasta que aparecen los síntomas. Lang et al., describen un caso en el que en el mismo paciente aparecen dos cuadros diferenciados de osteomielitis por Salmonella con distintas localizaciones en el miembro inferior, y con 17 años de diferencia ${ }^{7}$. Banky et al., presentan también un caso de reaparición de la osteomielitis en la misma localización 12 años después ${ }^{8}$. Por tanto, la infección por Salmonella suele cronificarse, siendo muy difícil su erradicación completa. 
Son pocos los casos descritos sobre una osteomielitis por Salmonella de localización metafisaria en paciente inmunocompetente que simule un tumor óseo, como por ejemplo el de Salem et al. ${ }^{1}$ aunque sí existen otros casos clínicos descritos similares como el de Charosky et al. en el que se presenta un caso de osteomielitis por Salmonella que simula un tumor de células gigantes ${ }^{9}$, o el de Sánchez et al., en el que se describe una osteomielitis por Salmonella que simula una displasia fibrosa monostótica ${ }^{10}$.

El diagnóstico adecuado de la osteomielitis por Salmonella es difícil, y tanto la exploración clínica como las pruebas de imagen (radiografías, TC o incluso RM) van a ser de gran importancia. No obstante, el diagnóstico definitivo lo obtendremos mediante un cultivo del material obtenido de la lesión ${ }^{2,4}$.

El tratamiento definitivo de las infecciones óseas por Salmonella es difícil, y normalmente requiere de extensos múltiples desbridamientos asociados a terapias de antibiótico intravenoso de larga duración. Es importante que la antibioterapia se inicie tras obtener los cultivos bateriológicos, y suele consistir en ampicilina, cefalosporinas de tercera generación, cloranfenicol, azitromicina o fluoroquinolonas, como el ciprofloxacino, que tiene la habilidad de penetrar en los macrófagos, lo cual es de gran utilidad en estos cuadros, y ha demostrado ser eficaz en el tratamiento de las infecciones óseas ${ }^{1-6}$.

\section{Conclusiones}

Cualquier lesión ósea que aparece en la zona metafisaria de los huesos largos, predominantemente alrededor de la rodilla, es susceptible de ser un tumor óseo, y por tanto debe ser descartado. No obstante, en ocasiones puede tratarse de infecciones por microorganismos atípicos, como la Salmonella.

La Osteomielitis por Salmonella en pacientes inmunocompetentes es una patología infrecuente que puede, además, simular una lesión neoplásica si se encuentra en una localización atípica como las metáfisis. El diagnóstico debe ser clínico, radiológico y analítico, aunque el diagnóstico definitivo será mediante cultivo. El tratamiento definitivo es el legrado extenso y relleno de la lesión, asociados a antibioterapia, y es necesario tener en cuenta que la lesión puede recidivar. 


\section{Bibliografía}

1. Salem KH. Salmonella osteomyelitis: A rare differential diagnostic in osteolytic lesions around the knee. Journal of Infection and Public Health 2014; 7:66-9.

2. Vynichakis G, Chandrinos M, Angelis S, Bogris E, Michelarakis JN. Salmonella Osteomyelitis of the Proximal Tibia in a Previously Healthy Adolescent: A Case Report. Cureus 2019; 11(9): e5672.

3. McAnearney S, McCall D. Salmonella Osteomyelitis: a case report. Ulter Med J 2015; 84(3):171-2.

4. Hashimoto K, Nishimura S, lemura S, Akagi M. Salmonella osteomyelitis of the distal tibia in a healthy woman. Acta Med Okayama 2018; 72(6):601-4.

5. Tonogai I, Hamada Y, Hibino N, Sato R, Henmi T, Sairyo K. Salmonella osteomielitis of the distal radius in a healthy young adult patient: report of a rare case and literature review. The Journal of Medical Investigation 2015; 62:97-9.

6. Balakumar B, Gangadharan S, Ponmudi N, Kumar S, Prakash JJ, Palocaren T. Atypical osteomyelitis and concurrent septic arthritis due to Salmonella in immunocompetent children. Journal of Clinical Orthopaedics and Trauma 2017; 8:293-7.

7. Lang R, Maayan MC, Lidor C, Savin H, Kolman S, Lishner M. Salmonella paratyphi C osteomyelitis: reports of two separate episodes 17 years apart. Scandinavian Journal of Infectious Diseases 1992; 24:793-6.

8. Banky JP, Ostergaard L, Spelman D. Chronic relapsing Salmonella osteomyelitis in an immunocompetent patient: case report and literature review. Journal of Infection 2002; 44(1):44-7.

9. Charosky CB, Marcove RC. Salmonella paratyphi osteomyelitis: report of a case simulating a giant cell tumor. Clinical Orthopaedics and Related Research 1974; 288:277-81.

10. Sanchez AA, Mazurek MT, Clapper MF. Salmonella osteomyelitis presenting as fibrous dysplasia. A case report. Clinical Orthopaedics and Related Research 1996; 330:185-9. 